\title{
Three-Dimensional Printing of Mitral Valve Models Using Echocardiographic Data: Does it Add to The Knowledge of Cardiology Fellow Physicians in Training?
}

\section{Ziad Bulbul ( $\square$ zb21@aub.edu.lb)}

American University of Beirut Medical Center https://orcid.org/0000-0002-0893-0435

\section{Issam El Rassi}

American University of Beirut Medical Center

\section{Lamya Atweh}

American University of Beirut Medical Center

\section{Ramsey F. Hamade}

American University of Beirut Medical Center

Hani Tamim

American University of Beirut

Fadi Bitar

American University of Beirut Medical Center

\section{Research}

Keywords: Echocardiography, Three-dimensional, mitral valve, printing, educational, cardiology trainees

Posted Date: February 4th, 2021

DOI: https://doi.org/10.21203/rs.3.rs-169357/v1

License: (c) (i) This work is licensed under a Creative Commons Attribution 4.0 International License.

Read Full License 


\section{Abstract}

Background: High fidelity three-dimensional Mitral valve models (3D MVM) printed from echocardiography are currently being used in preparation for surgical repair. We hypothesize that printed 3DMVM could have relevance to cardiologists in training by improving their understanding of normal anatomy and pathology.

Methods: Fifteen fellow physicians in pediatrics and adult cardiology training were recruited. 3D echocardiography video clips of 6 mitral valves (one normal and five pathological) were displayed and the fellows were asked to name the prolapsing segments in each. Following that, still images of 3D MVMs corresponding to the same MVs seen in the clip were presented on a screen and the participating physicians were asked to answer a comprehensive questionnaire aimed at assessing whether the 3D model has improved their understanding of valvular anatomy. Finally, a printed 3D MVM of each of the previously projected valves was handed out, and the same questionnaire was re-administered to identify any further improvement in the participants' perception of the anatomy.

Results: The correct diagnosis using the echocardiography video clip of the Mitral valve was attained by $45 \%$ of the study participants. Both pediatric and adult trainees, regardless of the year of training demonstrated improved understanding of the anatomy of MV's after observing the latter's model image, with an even further improvement in that understanding after they had seen and physically examined the printed model.

Conclusion: Printed 3D MVM has a beneficial impact on the understanding of MV anatomy and pathology compared to 3D-Echocardiography (3DE) images in cardiology trainees.

\section{Introduction}

Since its initial introduction in the 1980s, Three-dimensional (3D) printing of radiographic based imaging has gained momentum as a clinical tool in the medical field over the last few years. From an initial use for industrial work [1], 3D printing first made its debut in the field of clinical care in the 1990s with the manufacture of the first cranial bone model [2]. Its use has become widespread in various medical fields from bio-printing tissue organs [3] extending currently to patient-specific implants, prostheses, and replicated anatomical models for education and surgical planning [4,5]. Three-dimensional models of hearts with several congenital defects are currently available [2]. The diagnostic and educational values of such models as physical tools for training, especially in the setting of pre-operative planning, are gaining interest.

Although the clinical use of 3D Echocardiography has been around for more than two decades, its value in generating 3D models of cardiac structures has not been thoroughly investigated. Echocardiographic 3D data sets of a mitral valve [Fig. 1], obtained via the transesophageal window, have been shown to have enough spatial and temporal resolution for clinical use. However, this remains suboptimal for 3D 
printing. Hence, obtaining 3D prints from echocardiography data has not been fully perfected and is lagging other radiographic based images.

Many commercially available 3D rendering software are currently available. The rendered dynamic 3DE based images are an excellent adjunct to the conventional two-dimensional echocardiography for surgical repair. In addition to the rendered 3D echocardiographic image, a 3D geometrical Model [ Fig. 2] can be generated based on an echocardiographic image. The model can be used to perform several measurements that are crucial for the understanding of the structures of the MV and the planning of surgical intervention.

Projecting a 3D structure on a flat screen inevitably affects the viewer's depth perception. Unlike 3DE images, the MVM when printed in 3D, provides excellent depth while conserving details.

We hypothesize that tangible printed 3D models of MV [Fig. 3] could objectively improve the understanding, of the MV geometry and depth perception, for cardiologists in-training, further enhancing their understanding of normal valve anatomy and pathology.

\section{Methods}

This study is a cross sectional questionnaire-based educational study administered to cardiology physicians in training, between June 2017 and March 2018, at the American University of Beirut-Medical Center (AUBMC). The study was approved by the research Institutional Review Board of AUBMC. Cardiology fellows consented to be part of the study, and emphasis was placed on the fact that participation is voluntary and that they can withdraw from the study at any point in time if they chose to do so. Moreover, all data collected including videos, clinical models, and fellows' details (except for their year of training and subspecialty) were anonymous. Confidentiality was maintained throughout the study. Sixteen cardiology fellows at different levels of training (eleven adult cardiology and five pediatric cardiology) were recruited and completed the study.

Imaging of the MV and the acquisition of 3D data sets was completed using a (t7-1) transesophageal probe and (iE-33) echocardiography platforms (Philips, Eindhoven Netherlands). Rendered 3D images of the mitral valve were generated using QLAB software v.8 (Philips, Eindhoven Netherlands) and were saved as anonymous video clips, (Audio Video Interleave format, Microsoft, Washington, USA).

Native 3D data sets were imported into commercially available software (Image Arena, TomTec GmbH. Munich, Germany), where another, plug in semi-automated specialized software (MVA package 2.1) analyzes and tracks the mitral valve and generates a geometrical model of the valve to include the annulus, leaflets and coaptation line. Those models were saved in JPEG (Joint Photographic Experts Group) format and exported as stereolithographic files (stl).

Files of the models were printed, on a commercial Ultimaker $2+{ }^{\mathrm{R}} 3 \mathrm{D}$ printer (Ultimaker B.V. Watermolenweg, Netherlands). The fidelity of the resulting models was highly conserved, as they 
resembled the original echocardiographic model pictures.

A total of six (One normal and five pathological) valves were rendered in 3D and saved as video clips. For each 3D rendered MV, a 3D model was generated, saved as still image and printed in 3D. Pathological models included: two valves with single leaflet segment prolapse [Fig. 3:A1 and A2], two valves with at least two segments prolapse involving both leaflets and lack of leaflet coaptation [Fig. 3: B1 and B2], and a fifth valve, marked as valve $C$, demonstrated a flail segment. Valve D was the normal valve [Fig. 3].

To test our hypothesis, a PowerPoint (Microsoft incorporation- Washington, USA) based questionnaire was prepared. The questionnaire was specifically designed for this project to elicit whether the 3D printed model improved participant perception and knowledge about the MV [Fig. 4].

Movie clips of 3D echo of the $6 \mathrm{MVs}$ were initially projected to the study participants, after which they were asked to name the pathological segment(s) of the MV leaflets, if any existed. Following that, pictures of the 3D generated models of the same valves were sequentially projected on a flat screen. Participants were asked if that had added to their understanding of the valve regarding the following categories: (1) anatomy in general, (2) area and prolapse of the leaflets, and (3) shape of the annulus. Finally, the corresponding 3D printed MV models were handed over to the participants for tangible assessment. The same questionnaire was administered once again following their assessment of the printed model.

\section{Statistical analysis:}

Data were entered and analyzed using the Statistical Package for Social Sciences program (SPSS Inc, Chicago, IL). For each case, the number and percentage of fellows giving the correct answer and whether it improved their understanding of MV anatomy and pathology was calculated and presented as a mean and standard deviation. Each fellow was asked to assign a response to each of the four questions describing how a certain image modality (3D model or 3D printed respectively) improved their understanding of MV anatomy and pathology. The available answers were: 'extremely', 'very', 'moderately', 'slightly', and 'not at all'. For the presentation of the perception data, we have created three groups of responses. A negative perception as indicated by "not at all" and "slightly" answers, a neutral perception as indicated by "moderately" answer, and a positive perception as indicated by "very" and "extremely"', Percentages of negative, neutral and positive responses were calculated for each category in all 6 cases.

In order to assess the overall responses and compare outcomes between adult and pediatric fellows on one hand, and between junior (first-year trainees) and senior fellows (second and third year trainees) on the other; number and percentages of positive answers were compared and the difference in mean scores was calculated using the Mann-Whitney test, where $p$-value $<0.05$ was used to indicate statistical significance. 


\section{Results}

\section{1-Video Clips:}

Based on the video clips of the valves (Table 1), fourteen participants (87.5\%) identified the normal case correctly. Twelve (75\%) and seven (44\%) of them named the correct prolapsing segment in the two models with one pathological segment. However, six (37.5\%), four (25\%) and only one (6.3\%) participant identified correctly, all the pathological segments in the last three complex cases (with more than one prolapsing segment present).

Table 1

Number and percentages of the participants who made the correct diagnosis of pathological segment of mitral valve based on video clip. Total number of participants $=16$

\begin{tabular}{|lllllll|}
\hline Video of 3D MV* & Case 1 & Case 2 & Case 3 & Case 4 & Case 5 & Case 6 \\
\hline $\mathrm{N} * \star(\%)$ & $14(87.5 \%)$ & $7(43.8 \%)$ & $12(75.0 \%)$ & $6(37.5 \%)$ & $4(25.0 \%)$ & $1(6.3 \%)$ \\
\hline \multirow{2}{*}{ MV: mitral valve; ${ }^{* \star N}$ : number of participants with correct answer } & & \\
\hline
\end{tabular}

\section{2- Picture of the 3D Model:}

Following the projection of the picture of the 3D constructed model of the valve, $(44.8 \%),(41.7 \%),(40.6 \%)$ and $(51.6 \%)$ of the group agreed that the picture of the model improved their understanding of the general appearance of the MV, area of the valve, prolapsing segments and shape of the annulus respectively.

Among the junior fellows: (38.1\%) indicated that the picture of the 3D model improved their general understanding of the mitral valve anatomy; $(45.2 \%)$ stated that it improved their perception of the valve's area, (46.3\%) agreed it changed positively their understanding of the leaflet prolapse, and $40.5 \%$ agreed it improved their understanding of the shape of the annulus. This is in comparison to the senior fellows of whom (42.6), (44.4), (55.6) and (42.6) \% agreed that the picture of 3D model improved their understanding of the valve in general, it's area, prolapsing leaflets, and the shape of the annulus respectively. (Table 2) 
Table 2

The change in fellows' perception of mitral valve anatomy after examination of 3D printed model as compared to the picture of 3D model. P-value is calculated for the positive response

\begin{tabular}{|c|c|c|c|c|}
\hline \multicolumn{2}{|l|}{ All Trainees $(n=16)$} & \multirow{2}{*}{$\begin{array}{l}\text { Picture of 3D Model } \\
(\%)\end{array}$} & \multirow{2}{*}{$\begin{array}{l}\text { Print of 3D Model } \\
(\%)\end{array}$} & \multirow[t]{2}{*}{ p-value } \\
\hline & Response & & & \\
\hline \multirow[t]{3}{*}{ General appearance } & Negative & $(24.0)$ & (8.3) & \\
\hline & Neutral & $(35.4)$ & $(15.6)$ & \\
\hline & Positive & $(40.6)$ & $(76.0)$ & $<0.0001$ \\
\hline \multirow[t]{3}{*}{ Area of valve } & Negative & $(25.0)$ & (9.5) & \\
\hline & Neutral & $(30.2)$ & $(15.8)$ & \\
\hline & Positive & $(44.8)$ & $(74.7)$ & $<0.0001$ \\
\hline \multirow[t]{3}{*}{ Prolapsing segments } & Negative & $(25.3)$ & (5.3) & \\
\hline & Neutral & $(23.2)$ & $(15.8)$ & \\
\hline & Positive & $(51.6)$ & $(78.9)$ & $<0.0001$ \\
\hline \multirow[t]{3}{*}{ Shape of annulus } & Negative & $(22.9)$ & $(9.4)$ & \\
\hline & Neutral & $(35.4)$ & $(21.9)$ & \\
\hline & Positive & $(41.7)$ & $(68.8)$ & $<0.0001$ \\
\hline \multicolumn{5}{|l|}{ Junior trainees $(n=7)$} \\
\hline \multirow[t]{3}{*}{ General appearance } & Negative & (19.0) & $(2.4)$ & \\
\hline & Neutral & $(42.9)$ & $(23.8)$ & \\
\hline & Positive & $(38.1)$ & $(73.8)$ & $<0.0001$ \\
\hline \multirow[t]{3}{*}{ Area of valve } & Negative & (19.0) & $(2.4)$ & \\
\hline & Neutral & $(35.7)$ & $(22.0)$ & \\
\hline & Positive & $(45.2)$ & $(75.6)$ & $=0.001$ \\
\hline \multirow[t]{3}{*}{ Prolapsing segments } & Negative & (19.5) & $(4.9)$ & \\
\hline & Neutral & $(34.1)$ & $(19.5)$ & \\
\hline & Positive & $(46.3)$ & $(75.6)$ & $=0.001$ \\
\hline \multirow[t]{3}{*}{ Shape of annulus } & Negative & $(16.7)$ & $(4.8)$ & \\
\hline & Neutral & $(42.9)$ & $(35.7)$ & \\
\hline & Positive & $(40.5)$ & $(59.5)$ & $=0.02$ \\
\hline
\end{tabular}




\begin{tabular}{|c|c|c|c|c|}
\hline \multicolumn{2}{|l|}{ All Trainees $(n=16)$} & Picture of 3D Model & Print of 3D Model & p-value \\
\hline \multicolumn{5}{|l|}{ Senior trainees $(n=9)$} \\
\hline \multirow[t]{3}{*}{ General appearance } & Negative & $(27.8)$ & (13.0) & \\
\hline & Neutral & $(29.6)$ & (9.3) & \\
\hline & Positive & $(42.6)$ & $(77.8)$ & $<0.0001$ \\
\hline \multirow[t]{3}{*}{ Area of valve } & Negative & $(29.6)$ & $(14.8)$ & \\
\hline & Neutral & $(25.9)$ & (11.1) & \\
\hline & Positive & $(44.4)$ & $(74.1)$ & $<0.0001$ \\
\hline \multirow[t]{3}{*}{ Prolapsing segments } & Negative & $(29.6)$ & (5.6) & \\
\hline & Neutral & $(14.8)$ & (13.0) & \\
\hline & Positive & $(55.6)$ & (81.5) & $<0.0001$ \\
\hline \multirow[t]{3}{*}{ Shape of annulus } & Negative & $(27.8)$ & (13.0) & \\
\hline & Neutral & $(29.6)$ & (11.1) & \\
\hline & Positive & $(42.6)$ & (75.9) & $<0.0001$ \\
\hline \multicolumn{5}{|l|}{ Adult trainees $(n=11)$} \\
\hline \multirow[t]{3}{*}{ General impression } & Negative & $(6.7)$ & $(0.0)$ & \\
\hline & Neutral & $(26.7)$ & $(23.3)$ & \\
\hline & Positive & $(66.7)$ & $(76.7)$ & $=0.10$ \\
\hline \multirow[t]{3}{*}{ Area of valve } & Negative & $(6.7)$ & $(0.0)$ & \\
\hline & Neutral & $(23.3)$ & $(20.7)$ & \\
\hline & Positive & $(70.0)$ & (79.3) & $=0.06$ \\
\hline \multirow[t]{3}{*}{ Prolapsing segments } & Negative & $(10.3)$ & (3.4) & \\
\hline & Neutral & $(20.7)$ & $(17.2)$ & \\
\hline & Positive & $(69.0)$ & (79.3) & $=0.03$ \\
\hline \multirow[t]{3}{*}{ Shape of annulus } & Negative & $(6.7)$ & $(0.0)$ & \\
\hline & Neutral & $(26.7)$ & $(26.7)$ & \\
\hline & Positive & $(66.7)$ & (73.3) & $=0.10$ \\
\hline \multicolumn{5}{|l|}{ Pediatric Trainee $(n=5)$} \\
\hline General impression & Negative & $(31.8)$ & $(12.1)$ & \\
\hline
\end{tabular}




\begin{tabular}{|c|c|c|c|c|}
\hline \multicolumn{2}{|l|}{ All Trainees $(n=16)$} & \multirow{2}{*}{$\begin{array}{l}\text { Picture of 3D Model } \\
(39.4)\end{array}$} & \multirow{2}{*}{$\begin{array}{l}\text { Print of 3D Model } \\
(12.1)\end{array}$} & \multirow[t]{2}{*}{ p-value } \\
\hline & Neutral & & & \\
\hline & Positive & $(28.8)$ & $(75.8)$ & $<0.0001$ \\
\hline \multirow[t]{3}{*}{ Area of valve } & Negative & (33.3) & $(13.6)$ & \\
\hline & Neutral & (33.3) & $(13.6)$ & \\
\hline & Positive & (33.3) & $(72.8)$ & $<0.0001$ \\
\hline \multirow[t]{3}{*}{ Prolapsing segments } & Negative & (31.8) & $(6.1)$ & \\
\hline & Neutral & $(24.4)$ & $(15.2)$ & \\
\hline & Positive & $(43.8)$ & (78.7) & $<0.0001$ \\
\hline \multirow[t]{3}{*}{ Shape of annulus } & Negative & $(30.3)$ & $(13.6)$ & \\
\hline & Neutral & (39.4) & (19.7) & \\
\hline & Positive & (30.3) & $(66.7)$ & $<0.0001$ \\
\hline
\end{tabular}

\section{3- Inspection of Printed Model}

Inspection of printed model has improved the fellows understanding of the mitral valve in all 4 categories according to the percentages of the positive responses obtained: 1- general appearance of the valve (76\%); 2 - area of the valve (74.7\%); 3 - prolapsing segments (78.9\%) and 4 - shape of the annulus (68.8\%). When comparing fellows according to their level of training; senior fellows were more likely to agree that the 3D printed model was helpful in improving their understanding of the mitral valve in all categories when compared to their junior counterparts: $(77.8 \%)$ of senior fellows agreed the 3D printed model improved their understanding of the general appearance of the valve vs $(73.8 \%)$ for junior fellows $((p=$ $0.05)$. The results were the same regarding the shape of the annulus: $(75.9 \%)$ vs. $(59.5 \%)(p=0.01)$ and their understanding of the prolapsing segments: $81.5 \%$ vs $75.6 \%(p=0.03)$. However, there was no statistical difference between junior and senior fellows for whether the 3D printed model improved their understanding of the valve's area :75.6\% vs $74.1 \%(p=0.05)$. (Table 3$)$ 
Table 3

Comparing Percentages of the fellows' responses on the improvement in their perception of mitral valve following inspection the 3D model printed model, according to years of training. P-value is calculated for the positive response

\begin{tabular}{|c|c|c|c|c|}
\hline \\
\hline \multicolumn{2}{|l|}{ Printed model } & \multicolumn{3}{|c|}{ Level of training } \\
\hline & & Junior & Senior & $\mathrm{p}$-value \\
\hline \multirow[t]{3}{*}{ General Appearance } & Negative & $(2.4 \%)$ & $(13.0 \%)$ & \\
\hline & Neutral & $(23.8 \%)$ & $(9.3 \%)$ & \\
\hline & Positive & $(73.8 \%)$ & $(77.8 \%)$ & $=0.05$ \\
\hline \multirow[t]{3}{*}{ Area of valve } & Negative & $(2.4 \%)$ & $(14.8 \%)$ & \\
\hline & Neutral & $(22.0 \%)$ & $(11.1 \%)$ & \\
\hline & Positive & $(75.6 \%)$ & $(74.1 \%)$ & $=0.73$ \\
\hline \multirow[t]{3}{*}{ Prolapsing segments } & Negative & $(4.9 \%)$ & $(5.6 \%)$ & \\
\hline & Neutral & $(19.5 \%)$ & $(13.0 \%)$ & \\
\hline & Positive & $(75.6 \%)$ & $(81.5 \%)$ & $=0.03$ \\
\hline \multirow[t]{3}{*}{ Shape of annulus } & Negative & $(4.8 \%)$ & $(13.0 \%)$ & \\
\hline & Neutral & $(35.7 \%)$ & $(11.1 \%)$ & \\
\hline & Positive & $(59.5 \%)$ & $(75.9 \%)$ & $=0.01$ \\
\hline
\end{tabular}

\section{4- Picture vs Print of 3D Model}

When comparing the picture of the 3D model to its corresponding 3D print: the whole group perceived that the printed model had significantly improved their understanding of the mitral valve over the model picture in all four categories: $76 \%$ vs. $46 \%(p<0.0001)$ for the general appearance, $74.7 \%$ vs $44.8 \%(p<$ $0.0001)$ for the area, $78.9 \%$ vs $51.6 \%$ for prolapsing segments $(p<0.0001)$ and $69 \%$ vs $42 \%(p<0.0001)$ for shape of the annulus. (Table 2)

These results were the same regardless of the year of training; Junior fellows agreed the 3D printed model improved their understanding of the mitral valve in all four categories when compared to the picture of the 3D model: $73.8 \%$ vs $38.1 \%(p<0.0001)$ for general appearance, $75.6 \%$ vs $45.2 \%$ for the valve's area $(p<0.001), 75.6 \%$ vs $46.3 \%(p<0.001)$ for prolapsing segments : $59.5 \%$ vs $40.5 \%(p=0.02)$ for the shape of the mitral valve. Senior fellows followed the same trend: $77.8 \%$ vs $42.6 \%(p<0.0001)$ for the general appearance, $74.1 \%$ vs $44.4 \%(p<0.0001)$ for the area, $81.5 \%$ vs $55.6 \%(p<0.0001)$ for prolapsing segments, and $75.9 \%$ vs $42.6 \%(p<0.0001)$ for the shape of the annulus.

\section{Discussion}


The complexity of the mitral valve has been challenging to image ever since the initial stages of echocardiography. Advancement in 2DE during the last three decades; however, has revolutionized the cardiologists' understanding of the mitral valve over the initial " $\mathrm{B}$ " and " $\mathrm{M}$ " echocardiographic imaging modes. The introduction of the mono- and biplane electronic phased-array probes in 1982, on the other hand establishes a new a landmark in the clinical use of Trans Esophageal Echocardiograms (TEE) [6]. Two dimensional TEE and its application on the mitral valve imaging $[7,8]$ has expanded the scope of mitral valve repair surgery [9]. Before the introduction of 3D TEE, the combination of perioperative TEE data with the results of direct intraoperative inspection of the MV in an arrested heart was the standard practice surgeons used in making decision regarding mitral valve repair. Despite all advancement, the understanding of the MV pathology requires the assimilation of two-dimensional images with direct intraoperative valve findings. Ahmed et al reported that this approach could be challenging for less experienced echocardiographers; especially in cases of complex MV prolapse involving multiple MV segments [10].

Training a cardiologist on echocardiography is a tedious and lengthy process. The biggest challenge for a trainee is to mentally reconstruct a 3D image from multiple slices of 2D planes of a given structure. To achieve such purpose, the advantage of 3DE over 2D TEE is obvious, especially for a complex structure like the MV. Furthermore, La Canna et al. eluded to an additional diagnostic value of 3D TEE, in that it provides more accurate mapping of MV prolapse and further details of MV anatomy [11].

Currently, three-dimensional images rendered from 3DE are projected on 2D monitors. The mere fact of projecting a three-dimensional structure on a flat screen is a significant shortcoming, despite the use of different hues and shadows to improve depth perception.

Recently, 3D printing has gained momentum in the medical field. Anatomical structures from different systems of the body are printed in a very high degree of accuracy $[4,5]$. Moreover, the price of printing has decreased significantly, and most centers nowadays can print their 3D images.

While printing cardiac structures from CT and MRI scans are done routinely nowadays; printing from echocardiographic data has not been perfected yet. This is mainly due to the relatively low special resolution of 3DE images. Mahmoud et al [11] and others have printed 3D models of the mitral valve generated from echocardiographic data. The resulting models closely mimicked the original echocardiographic pictures. This imaging modality however still requires some refinement to better describe leaflet coaptation, chordae, papillary muscles and left ventricular geometry [13].

In the field of surgery, the improvement in MV surgical outcomes coinciding with the use of 3D based images was highlighted by Beraud and his group [14]. Hien et al. had shown in a multicenter study that the use of 3DE of the Mitral valve improves the understanding of prolapse segments [15]. The fact that this was true for beginners and experienced echocardiographers alike was of interest. Taking it one step further, Hadeed and his group in a brief communication, proposed the use of printed 3D models of complex congenital lesions as a roadmap for surgical repair [16]. Recently, Premyodhin et al. published 
their experience in the use of a specially constructed MV molds, that simulated natural tissue in the preoperative simulation of robotic mitral valve repair [17].

From the educational point of view; Biglino et al, in a recent publication showed the benefit of using 3D models of the heart as an educational tool for the training of both adult and pediatric cardiac nurses [18]. Costello et al. found that the even pediatric residents' understanding of lesions like ventricular septal defect could be further enhanced by the printed model as compared to educational seminars in anatomy and echocardiography. They proposed that 3D printed heart models can be effectively incorporated into a simulation-based congenital heart disease and critical care training curriculum for pediatric resident physicians [19].

One of the advantages of the physical inspection of the 3D model over a 2D projection of its image could be explained by the loss of the depth perception that is intrinsic to projecting a 3D structure onto a $2 \mathrm{D}$ screen.

In this study, we sought to prove that a physical model of the mitral valve, printed based on MV echocardiographic data, can enhance the understanding of the normal and abnormal anatomy of mitral valves even at a high level of training. Our data clearly shows that the MV model, when physically examined by the cardiology fellows, did significantly improve their understanding even after seeing a 3D image of the same model. This observation was not only valid for the prolapsing segments but also for the area and shape of the valve, and the complex anatomy of the annulus. This was the case irrespective of the level of training of the cardiology fellow.

In the future, further advancement in the spatial and temporal resolution of echo generated 3D images along with the advancement in 3D printing, will permit the printing of actual structures of normal as well as congenitally deformed hearts from echocardiographic data in high fidelity and accuracy. Eventually, combining valves and subvalvular apparatus printed from echocardiographic data with heart models printed from CT or MRI scans will further improve our understanding of congenital heart disease as well as surgical planning.

\section{Study Limitations:}

Although the number of study participants was small, the results were coherent for the superiority of the 3D printed model. This study could thus serve as a starting point for larger studies to assess the effectiveness of $3 \mathrm{D}$ models integration in medical education.

\section{Conclusion}

Three-dimensional models of MV manufactured based on echocardiographic data and printed by means of 3D technology have a positive impact on the understanding of adult and pediatric cardiology trainees, of the MV anatomy and pathology. It might further enhance the diagnostic power of 3DE especially in complex cases of MV disease.

Page $11 / 18$ 


\section{Abbreviations}

2D: Two-dimensional

3D: Three-dimensional

3D MVM: Three-dimensional mitral valve models

3DE: Three-dimensional echocardiography

AUBMC: American University of Beirut-Medical Center.

TEE: Trans esophageal echocardiograms

\section{Declarations}

\section{Ethics approval and consent to participate}

This study was approved by the Institutional Review Board (IRB) and the ethics committee at the American University of Beirut Medical Center and AUB Faculty of Medicine. Consent was obtained from all fellows (pediatric and adult cardiology) prior to enrollment. Enrollment was optional and participation was solely up to the fellow choosing.

\section{Consent for Publication}

Not applicable.

\section{Availability of data and material}

The datasets generated and/or analyzed during the current study are available from the corresponding author on reasonable request

\section{Conflicts of interest/ Competing Interests}

The authors declare they have no competing interests

\section{Funding}

All 3D models were printed in department of Engineering at the American University of Beirut by Dr. Hamadeh and his group, funded by the National Council for Scientific Research (Grant Research Program 2017 award number 103288).

\section{Ethics approval and consent to participate}

Not applicable 


\section{Acknowledgments}

We would like to thank Miss Farah Tibni for helping with the printing of the 3D models and Mr. Amer Bulbul for helping with data entry and preparation of the figures

\section{Author's Information}

ZIAD BULBUL, MD is an associate professor of Medicine at the American University of Beirut Faculty of Medicine in the department of Pediatrics and Adolescent Medicine. He is the principal investigator of the study. He is program director for the pediatric cardiology fellowship

ISSAM EL RASSI, MD, is an assistant professor of Surgery at the American University of Beirut Faculty of Medicine specialized in Congenital Pediatric Heart Surgery. He is also the Co-Founder of the "Heart Beat Fund", a charitable initiative providing financial and assistance to needy families suffering from Congenital Heart Disease.

LAMYA ANN ATWEH. Assistant Professor of Diagnostic Radiology at the American University of Beirut Faculty of Medicine. She is a pediatric attending radiologist at the American University of Beirut Medical Center

RAMSEY HAMADE, PhD is a Professor of Engineering at the Maroun Semaan Faculty of Engineering and Architecture in the Department of Mechanical Engineering. He is responsible for the generation of the 3D printed models of the heart valves at the Department of Engineering at the American University of Beirut

HANI TAMIM, PhD is an associate professor at the American University of Beirut Faculty of Medicine in the department of Internal Medicine. He is the leading statistician on the paper and was responsible of data collection, cleaning and analysis.

FADI BITAR, MD Professor of Pediatrics, Biochemistry and Molecular Genetics, Founding Director of the Children's Heart Center and Associate Dean for External Medical Affairs at the American University of Beirut, Lebanon. He is President of the Pan Arab Congenital Heart Disease Association and Co-founder of the "Brave Heart Fund".

\section{References}

1. Sun Z, Squelch A. 3D printed models of complex anatomy in cardiovascular disease. Heart Research Open Journal. 2015;2(3):103-8.

2. Kim MS, Hansgen AR, Wink O, Quaife RA, Carroll JD. Rapid prototyping: a new tool in understanding and treating structural heart disease. Circulation. 2008;117(18):2388-94.

3. Daemen JH, Heuts S, Olsthoorn JR, Maessen JG, Sardari Nia P. Mitral valve modelling and threedimensional printing for planning and simulation of mitral valve repair. European Journal of CardioThoracic Surgery. 2019; 55(3):543-551. 
4. Frame M, Leach W. DIY 3D printing of custom orthopaedic implants: a proof of concept study. Surgical technology international. 2014; 24:314-7.

5. Gerstle TL, Ibrahim AM, Kim PS, Lee BT, Lin SJ. A plastic surgery application in evolution: threedimensional printing. Plastic and reconstructive surgery. 2014;133(2):446-51.

6. Souquet J, Hanrath P, Zitelli L, Kremer P, Langenstein B, Schluter M. Transesophageal phased array for imaging the heart. IEEE transactions on Biomedical Engineering. 1982(10):707-12.

7. Lambert A-S, Miller JP, Merrick SH, Schiller NB, Foster E, Muhiudeen-Russell I, et al. Improved evaluation of the location and mechanism of mitral valve regurgitation with a systematic transesophageal echocardiography examination. Anesthesia \& Analgesia. 1999;88(6):1205-12.

8. Shanewise JS, Cheung AT, Aronson S, Stewart WJ, Weiss RL, Mark JB, et al. ASE/SCA guidelines for performing a comprehensive intraoperative multiplane transesophageal echocardiography examination: recommendations of the American Society of Echocardiography Council for Intraoperative Echocardiography and the Society of Cardiovascular Anesthesiologists Task Force for Certification in Perioperative Transesophageal Echocardiography. Journal of the American Society of Echocardiography. 1999;12(10):884-900.

9. Sidebotham DA, Allen SJ, Gerber IL, Fayers T. Intraoperative transesophageal echocardiography for surgical repair of mitral regurgitation. Journal of the American Society of Echocardiography. 2014;27(4):345-66.

10. Ahmed S, Nanda NC, Miller AP, Nekkanti R, Yousif AM, Pacifico AD, et al. Usefulness of Transesophageal Three-Dimensional Echocardiography in the Identification of Individual Segment/Scallop Prolapse of the Mitral Valve. Echocardiography. 2003;20(2):203-9.

11. La Canna G, Arendar I, Maisano F, Monaco F, Collu E, Benussi S, et al. Real-time three-dimensional transesophageal echocardiography for assessment of mitral valve functional anatomy in patients with prolapse-related regurgitation. American Journal of Cardiology. 2011;107(9):1365-74.

12. Mahmood F, Owais K, Taylor C, Montealegre-Gallegos M, Manning W, Matyal R, et al. Threedimensional printing of mitral valve using echocardiographic data. JACC Cardiovascular imaging. 2015;8(2):227-9.

13. Witschey WR, Pouch AM, McGarvey JR, Ikeuchi K, Contijoch F, Levack MM, et al. Three-dimensional ultrasound-derived physical mitral valve modeling. The Annals of thoracic surgery. 2014;98(2):691-4.

14. Beraud A-S, Schnittger I, Miller DC, Liang DH. Multiplanar reconstruction of three-dimensional transthoracic echocardiography improves the presurgical assessment of mitral prolapse. Journal of the American Society of Echocardiography. 2009;22(8):907-13.

15. Hien MD, Großgasteiger M, Rauch H, Weymann A, Bekeredjian R, Rosendal C. Experts and beginners benefit from three-dimensional echocardiography: a multicenter study on the assessment of mitral valve prolapse. Journal of the American Society of Echocardiography. 2013;26(8):828-34.

16. Hadeed K, Dulac Y, Acar P. Three-dimensional printing of a complex CHD to plan surgical repair. Cardiology in the Young. 2016;26(7):1432-4. 
17. Premyodhin N, Mandair D, Ferng AS, Leach TS, Palsma RP, Albanna MZ, et al. 3D printed mitral valve models: affordable simulation for robotic mitral valve repair. Interactive cardiovascular and thoracic surgery. 2017;26(1):71-6.

18. Biglino G, Capelli C, Koniordou D, Robertshaw D, Leaver LK, Schievano S, et al. Use of 3D models of congenital heart disease as an education tool for cardiac nurses. Congenital heart disease. 2017;12(1):113-8.

19. Costello JP, Olivieri LJ, Su L, Krieger A, Alfares F, Thabit O, et al. Incorporating three-dimensional printing into a simulation-based congenital heart disease and critical care training curriculum for resident physicians. Congenital heart disease. 2015;10(2):185-90

\section{Figures}

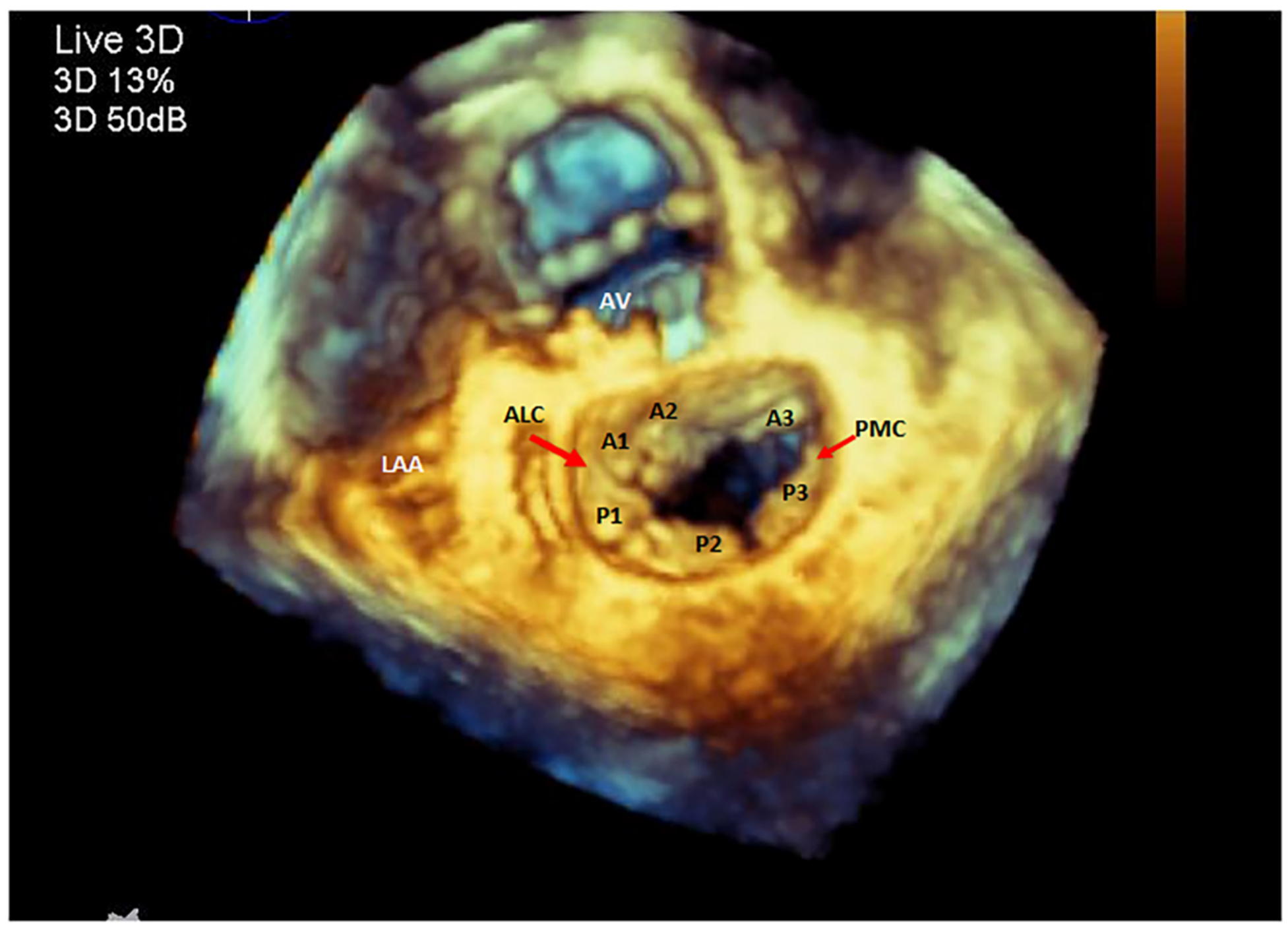

\section{Figure 1}

Three-dimensional Echocardiography image of the mitral valve showing the normal shape of the annulus, both leaflets and the corresponding segments. (LAA: left atrial appendage, ALC: Antero-lateral commissure, PMC: poster0-medial commissure.) 


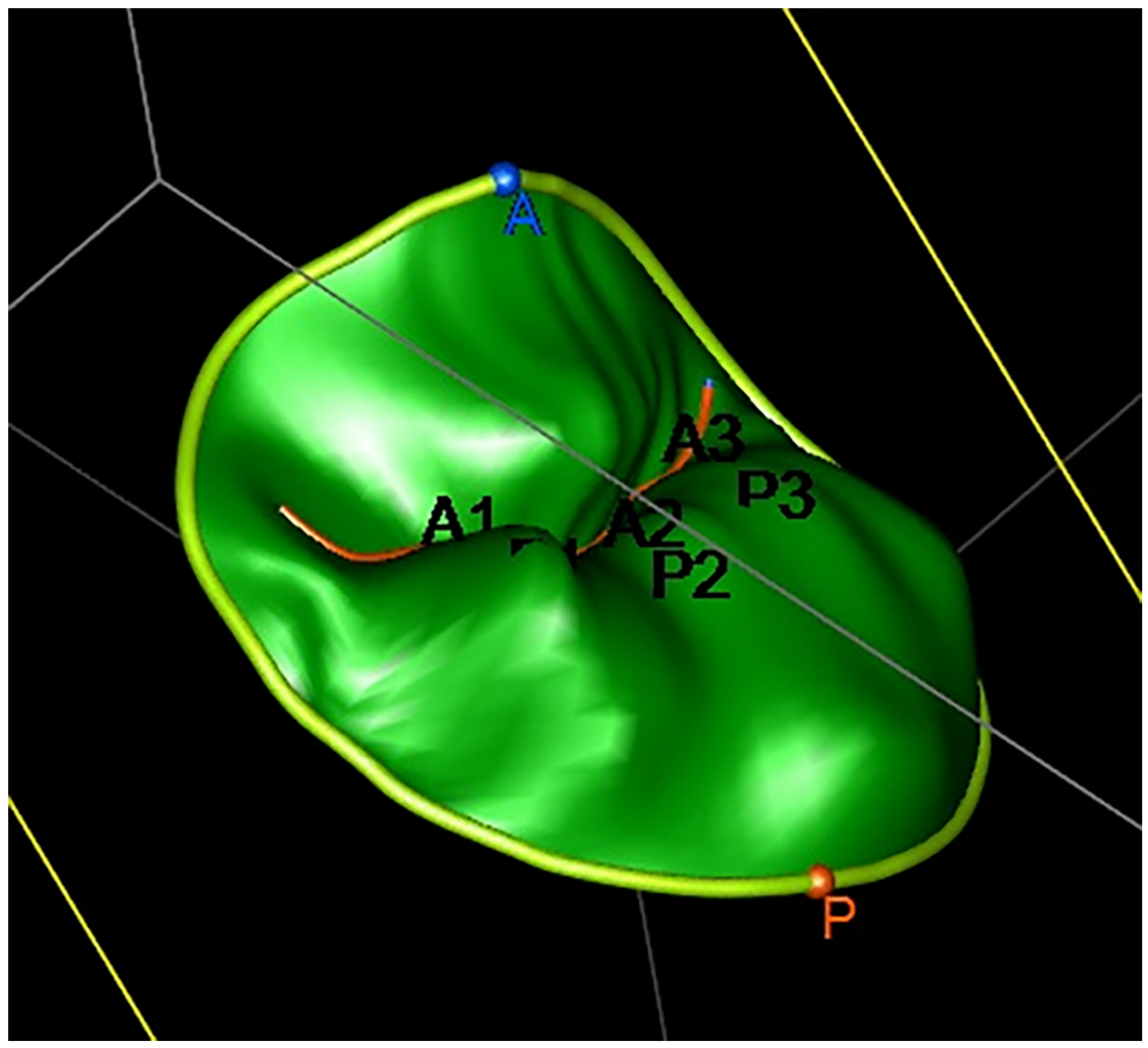

Figure 2

The two leaflets are divided into a total of six scallops: A1, A2, A3 (anterior) and P1, P2, P3 (posterior) 


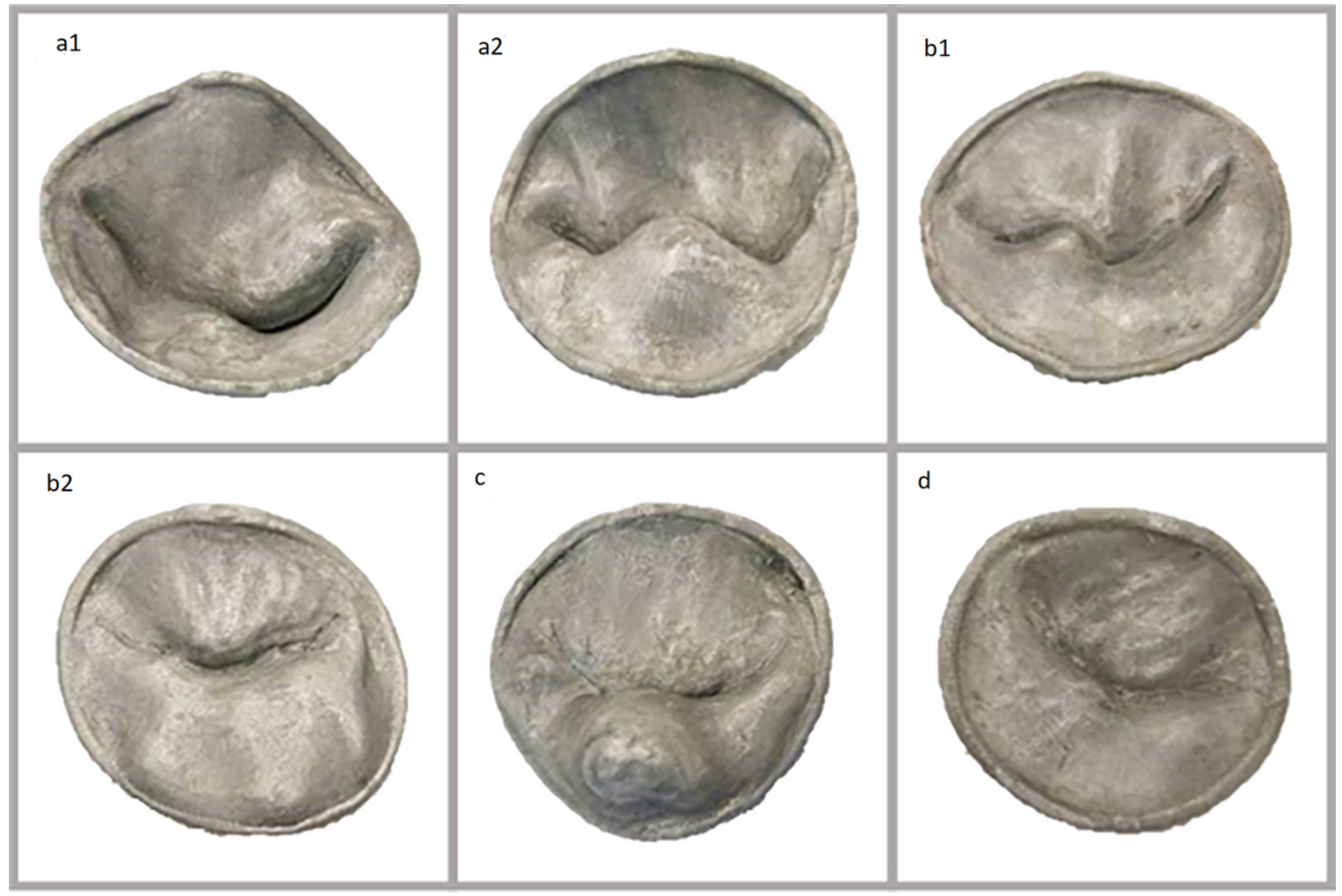

Figure 3

(a1) and (a2): two valves with single leaflet segment prolapse; (b1) and (b2): two valves with at least two segments prolapse involving both leaflets, with lack of leaflet coaptation in one. (c): valve with a flail segment. (d): normal valve 


\section{Case......}

\section{D Video clip}

Which MV segment/§.is/are prolapsing?

\begin{tabular}{|c|c|c|}
\hline A1 & P1 & Ine \\
\hline $\mathrm{A} 2$ & P2 & \\
\hline A3 & $\mathrm{P}_{3}$ & \\
\hline
\end{tabular}

\section{D Model Image}

Did the model add to your understanding of the DaX

- In General:

- Regarding: Area and configuration of the leaflets:

$\square$ Not at all $\square$ Sightly

- Prolapsing segments:

- Shape and spatial configuration of the annulus:

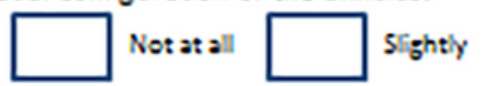

\section{D PRINTED Model}

Did the model add to your understanding of the Bay

- In General:

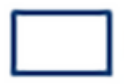
Not at a
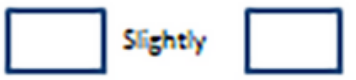

Moderately

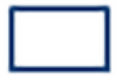

Very

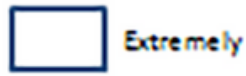

- Regarding: Area and configuration of the leaflets:

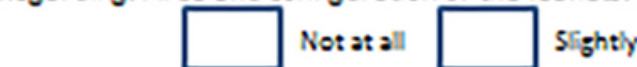

Sightly

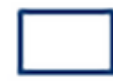

Moderately

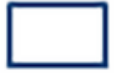

Very

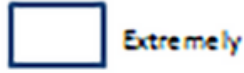

- Prolapsing segments:
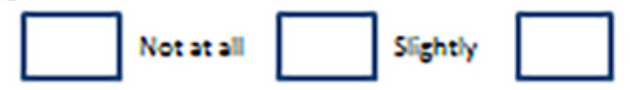

Moderately

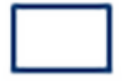

Very

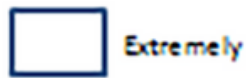

- Shape and spatial configuration of the annulus:

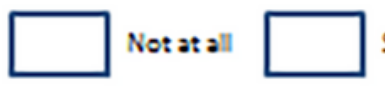
Slghtly

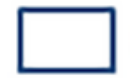

Moderately

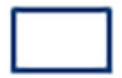

Verv

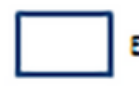

Extremely

\section{Figure 4}

Questionnaire designed to elicit whether the 3D printed model improved participant perception and knowledge about the mitral valve 